Spyrou E.D., Anagnostopoulou A., Stylios C. (2021) DSRC or LTE? Selecting the Best Medium for V2I Communication Using Game Theory. In: Camarinha-Matos L.M., Boucher X., Afsarmanesh H. (eds) Smart and Sustainable Collaborative Networks 4.0. PRO-VE 2021. IFIP Advances in Information and Communication Technology, vol 629. Springer, Cham. https://doi.org/10.1007/978-3-030-85969-5_54

\title{
DSRC or LTE? Selecting the Best Medium for V2I Communication using Game Theory
}

\author{
Evangelos D. Spyrou ${ }^{1}$, Afroditi Anagnostopoulou ${ }^{2}$, Chrysostomos Stylios ${ }^{1}$ \\ ${ }^{1}$ Laboratory of Knowledge and Intelligent Computing, Department of Informatics and \\ Telecommunications, University of Ioannina, stylios@uoi.gr \\ ${ }^{2}$ Hellenic Institute of Transport, Centre for Research and Technology Hellas
}

\begin{abstract}
Vehicular communication is a very challenging and essential research area capable of supporting safety and routing decision-making. Vehicle to Infrastructure (V2I) communication often refers to communication between vehicles and Road Side Units (RSU), and recently several technologies have been developed to support it, such as ZigBee, Wi-Fi, GSM, Long Term Evolution (LTE), and 802.11p Direct Short Range Communication (DSRC). In this field, there is a competition between wireless DSRC and cellular LTE to define the most efficient type of communication. This paper aims to analyze the strengths and weaknesses of the DSRC and LTE to evaluate their performances and select the right technology for communication between vehicles and RSUs. Therefore, a vehicle equipped with both LTE and DSRC modules is assumed, and we propose a game-theoretic formulation to select the most efficient type of communication. The proposed formulation results in two equilibria; based on them, the vehicle and the RSU select the same communication module. Here it presents the correlated equilibrium when a trusted source makes the decision, and it discusses the two equilibria as a potential game formulation.
\end{abstract}

Keywords: LTE, DSRC, RSU, vehicle, game theory,

\section{Introduction}

The automotive industry offers an enormous testbed for new technologies such as vehicular communication, supporting safety, and routing decision-making in the modern environment. Many sensors are embedded in vehicles, and information is exchanged among them, establishing the Vehicle to Vehicle (V2V) communication. Moreover, Vehicle to Infrastructure (V2I) utilizes Road Side Units (RSU) to exchange information, which paves the way for new services.

V2I communication is essentially the exchange of messages or data between a vehicle and an RSU. V2V communication is an ad-hoc network in which vehicles create a Vehicular Ad-hoc Network (VANET), and when two vehicles are within range with each other, they transfer data, or they encapsulate multihop communication $[1,2]$. In other words, VANET is a form of wireless network, which includes mobility in conjunction with the other properties of the wireless channel. Multiple RSUs, hotspots, 
and cellular base stations are deployed in the road network. The network infrastructure includes wired devices such as routers or switches and cloud formations [3] or fog computing [4] devices.

Several technologies are available for V2I communication, including ZigBee, GSM [5], DSRC, Wi-Fi [6], LTE, and LTE-V2V [2]. In this field, a competition between wireless DSRC (i.e., $802.11 \mathrm{p}$ protocol) and cellular LTE (i.e., 4G telecommunications) has been established to define the most efficient type of V2I communication. If a vehicle includes both technologies in a dual-mode, the most efficient selection is based on the current conditions in the network, such as density, interference, and distance.

This paper aims to analyze the strengths and weaknesses of the DSRC and LTE to evaluate their performances and select the right technology for communication between vehicles and RSUs, which are available at traffic lights. The proper technology should be selected to communicate between vehicles and traffic lights to transfer their data and information. Note that LTE can be substituted by a 5G telecommunication system [7] since it will offer less latency and higher throughput, but this is beyond the scope of this paper since $4 \mathrm{G} / \mathrm{LTE}$ is a standard technology for vehicular networks, we selected it for this work.

Here, we propose applying a game-theoretic model, whereby the vehicle and the traffic light device must select the same technology. The proposed method reaches an equilibrium of the game, where a central planner chooses the players with respect to the type of messages that will get exchanged. Furthermore, it is proved that this is a potential game that has two Nash equilibria. Finally, it is also proved that the fictitious game learning converges to the two Nash equilibria.

More specifically, in this paper, we show the following contributions:

- We suggest a game-theoretic model for selecting the same network (LTE or DSRC) between a traffic light and a passive vehicle for data transmission.

- We show a correlated equilibrium when a trusted centralized planner selects the choice for both players.

- We propose a modified game as the model for our solution.

- We show that this game is a potential game, which means that it converges to a Nash equilibrium.

- This game has two Nash equilibria in the pure strategies.

The remainder of this paper is as follows: section 2 briefly discusses LTE and DSRC technologies, section 3 provides a brief comparison of the two technologies, section 4 gives a background on game theory and potential games, section 5 describes the derivation of the game-theoretic model, section 6 gives the results and in section 7 conclusions are provided.

\section{DSRC and LTE}

In this section, we provide a summary of the LTE and DSRC technologies for vehicular communications. Our purpose is to show the critical points of each communication medium. 


\subsection{DSRC}

Vehicular wireless communication evolved with the emergence of the IEEE $802.11 \mathrm{p}$ standard, which results in DSRC. The environment where V2X devices are deployed is quite tricky since interference is high due to buildings infrastructure or other frequencies that act in the area. The DSRC medium offers $75 \mathrm{MHz}$ broadband communication in the 5.850 - 5925 frequencies [8]. Moreover, DSRC offers high data rates for V2I and V2V communications. The DSRC standard embeds the IEEE 1609.x protocol family and the $802.11 \mathrm{p}$. In particular, the IEEE 1609 resides on top of IEEE $802.11 \mathrm{p}$. It enables the operation of the upper layers from the physical layer and the access control of the MAC layer, across multiple channels, without having information regarding parameters of low-level layers. [9].

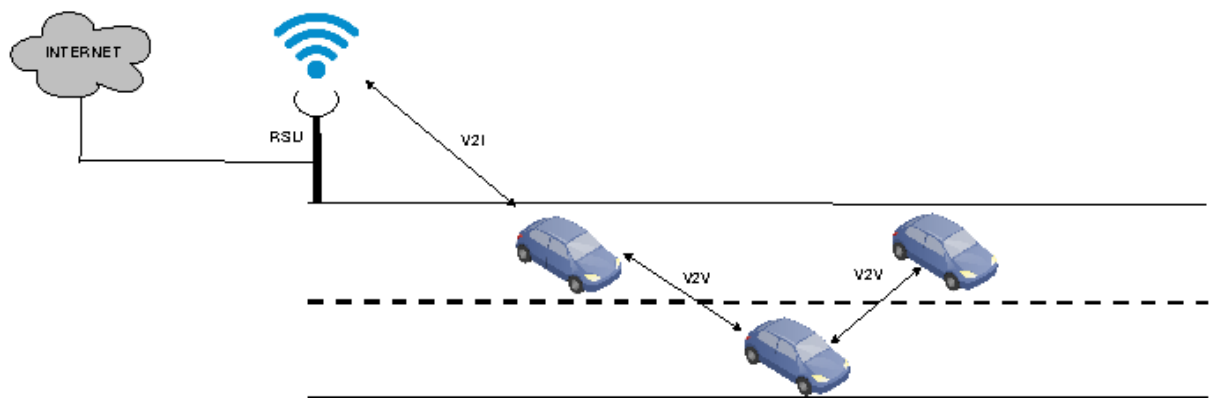

Fig. 1: DSRC Example

At the PHY layer, DSRC utilizes orthogonal frequency division multiplexing (OFDM) with convolutional coding. The IEEE $802.11 \mathrm{p}$ operates in a $10 \mathrm{MHz}$ bandwidth channel and uses doubled OFDM symbol duration and guard interval to counter larger delay spreads. It results in the subcarrier spacing that is halving to 156.25 $\mathrm{kHz}$. Furthermore, IEEE $802.11 \mathrm{p}$ introduces improved receiver performance requirements in adjacent channel rejections, handling cross-channel interference. There are defined four spectrum masks utilized in different operations and are more stringent than those demanded of the IEEE 802.11 radios. As for the MAC layer, IEEE $802.11 \mathrm{p}$ uses Carrier Sensing Multiple Access with Collision Avoidance (CSMA/CA). In order to improve latency of the Basic Service Set (BSS) procedure, the standard introduces the Wave BSS, whereby vehicles transmit signals without prior association, speeding up the whole process. In order to overcome packet collisions, decentralized congestion control techniques are used, with which adaptation of transmissions concerning congestion of the channel is promoted [10]. Fig 1 presents the DSRC V2X communication structure.

\subsection{LTE}

The LTE standard by the 3rd Generation Partnership Project (3GPP) [11] has come into play, offering excellent performance in throughput and latency. The access network consists of the eNodeBs and the user equipment (UE). The eNodeBs act as centralized 
base stations, containing a packet programmer, which selects the traffic rate depending on service demands. In theory, LTE can reach a $150 \mathrm{Mbps}$ downlink data rate and a 50 Mbps uplink data rate. In combination with latency less than $5 \mathrm{~ms}$ in the user plane, LTE is a strong candidate for vehicular communications.

Moreover, LTE uses orthogonal frequency division multiple access (OFDMA) for downlink and Single Carrier Frequency Division Multiple Access (SC-FDMA) for uplink connections. The width of the channel is from 1.4 to $20 \mathrm{MHz}$. LTE also supports MIMO giving an advantage in dynamic conditions, such as vehicular applications.

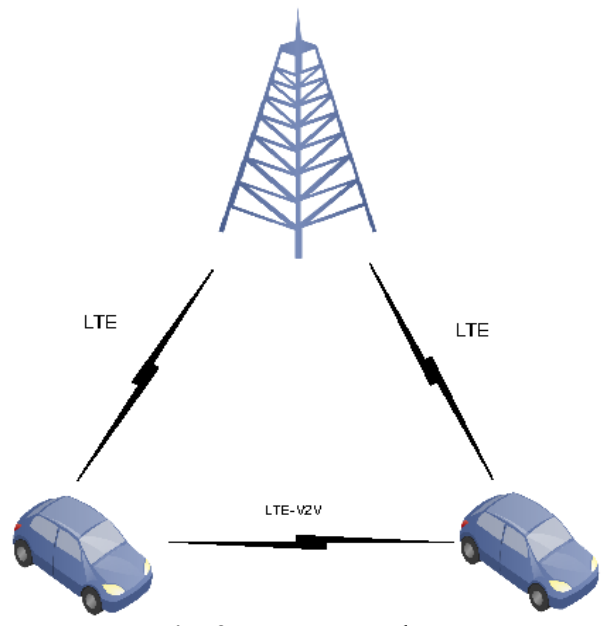

Fig. 2: LTE Example

The eNodeBs comprise the radio access network (RAN) of the LTE network architecture, responsible for radio control and management functionalities and the communication between the UEs and the LTE core. The eNodeBs relate to the Evolved Packet Core (EPC), which can take care of mobility management quality of service and interoperability with legacy $3 \mathrm{GPP}$ or other technologies [12]. An amendment of the LTE is the LTE-A, which offers a more significant bit rate, capacity, and spectrum utilization [13]. Lastly, LTE may offer direct communication between UEs, like DSRC communication, which does not require a base station for its operation. The LTE standard provides broadband communication and enables vehicular applications either by using On-Board Units (OBU) or by smartphone LTE connectivity. Fig. 2 presents an essential LTE communication.

\section{LTE and DSRC Comparison}

There is a competition between wireless DSRC and cellular LTE, which defines the most efficient type of communication among vehicular applications. Several research 
works attempt to find the proper answer. DSRC has been installed in USA cities even though there is an issue with its reliability and efficiency, particularly in high vehicle density applications [14]. The DSRC radio spectrum requires high data traffic demand for in-vehicle Internet access. On the other hand, cellular approaches, such as LTE, offer high capacity, broad coverage, range, and widely existing infrastructure. The main issue with cellular approaches is the centralized means of communication, which may cause problems in the latency between $\mathrm{V} 2 \mathrm{~V}$ communications. The support of distributed resource management is a significant problem, which is a requirement for allowing V2V operation in the absence of a network infrastructure. Latency may also appear, which is a severe drawback, especially for safety-critical applications. Moreover, a potential problem with using cellular approaches, such as LTE, is the accommodation of $\mathrm{V} 2 \mathrm{X}$ data traffic and the increasing data traffic from its legacy users.

Mir and Filali [12] evaluated the IEEE 802.11p and LTE in terms of delay, reliability, scalability, and mobility using different networking conditions and settings. LTE exhibits better network capacity and supports in-vehicle mobility than IEEE $802.11 \mathrm{p}$. Concerning the transmission delays, the authors pointed out that the delay increases with the increase of the network load. On the other hand, IEEE $802.11 \mathrm{p}$ exhibited acceptable performance when there is mobility support, and the topologies are sparse. It identifies that IEEE $802.11 \mathrm{p}$ performance decreases when it suffers from large vehicle density or traffic load. LTE includes infrastructure-oriented scheduling and access control, as well as it does not contain a vast number of network elements; hence, its performance may surpass the IEEE $802.11 \mathrm{p}$.

Theoretical work shows that in safety-critical vehicular applications, beaconing in LTE is poor due to network overload, even in idealistic conditions [15]. Therefore, the DSRC architecture indeed appears more promising in safety-critical vehicular applications. Based on [16], a combination of the two technologies is the best option. In particular, LTE wins in terms of capacity and communication range, and it does not miss any beacons due to collisions. On the other hand, $802.11 \mathrm{p}$ accomplishes better latency due to direct communication.

Overall, the performance of IEEE 802.11p and LTE depend on the conditions even though LTE could be characterized as a better choice in most of the studied cases. Therefore, we may consider "infrastructure" and "passing vehicle" as players that want to select the best communication medium for their data transmission in the proposed methodology on game theory.

\section{Game Theory and Potential Games}

Game theory studies mathematical models of conflict and cooperation between players [17]. The meaning of the term game corresponds to any form of interaction between two or more nodes. The rationality of a node is satisfied if it pursuits the satisfaction of its preferences through the selection of appropriate strategies. The preferences of a node need to satisfy general rationality axioms, and then its behavior can be described by a utility function. Utility functions provide a quantitative description of the node's preferences, and the main objective is to maximize its utility function.

In this work, we propose strategic non-cooperative games since we consider nodes to act as selfish players that want to preserve their interests. The intuition behind this is 
that the nodes will reach an optimal state without paying the price to maximize their payoffs. The Nash equilibrium [18] is the most crucial equilibrium in non-cooperative strategic form games. It is defined as the point where no node will increase its utility by unilaterally changing its strategy.

In 2008, Daskalakis proved that finding a Nash equilibrium is PPAD-complete [19]. Polynomial Parity Arguments on Directed graphs (PPAD) is a class of total search problems [20] for which solutions have been proven to exist. However, finding a specific solution is difficult if not intractable. This development drove the community to 'Potential Games' [21] since they guarantee the convergence to pure Nash equilibria and best response dynamics.

This class of games consists of the exact, ordinal potential and weighted. This work employs weighted potential games. For the sake of clarity, we mention the necessary conditions for games to be classified as potential. More formally:

A game $\Gamma\langle N, A, u\rangle$, with $N$ players, $A$ strategy profiles and $u$ the payoff functions, is an exact potential game if there exists a potential function

$$
V: A \rightarrow \mathbb{R},(1)
$$

subject to

$$
\forall i \in N, \forall x_{-i} \in A_{-i}, \forall x_{i}, x_{i}^{\prime} \in A_{i}(2)
$$

Where $x_{i}$ is the strategy of player $i, x_{i}^{\prime}$ is the deviation of player $i, x_{-i}$ is the set of strategies followed by all the players except player $i$ and $A_{-i}$ is the set of strategy profiles of all players except $i$ such as

$$
V\left(x_{-i} x_{i}\right)-V\left(x_{-i}, x_{i}^{\prime}\right)=u\left(x_{-i}, x_{i}\right)-u\left(x_{-i}, x_{i}^{\prime}\right)
$$

In terms of an ordinal potential game, the necessary condition for its existence is

$$
V\left(x_{-i}, x_{i}\right)-V\left(x_{-i}, x_{i}^{\prime}\right)>0 \Leftrightarrow u\left(x_{-i}, x_{i}\right)-u\left(x_{-i}, x_{i}^{\prime}\right)>0
$$

A game $\Gamma$ is a weighted potential game [21] if there exists a vector of positive numbers $w=\left(w_{1}, \ldots, w_{n}\right) \in \mathbb{R}_{++}^{2}$ and a real-valued function $V: A \rightarrow R$ is a weighted potential if for every $i \in N$ and for every $x_{-i} \in A_{-i}$, for every $x_{i}, x_{i}^{\prime} \in A^{i}$

$$
u\left(x_{-i}, x_{i}\right)-u\left(x_{-i}, x_{i}^{\prime}\right)=w_{-} i\left(V\left(x_{-i}, x_{i}\right)-V\left(x_{-i}, x_{i}^{\prime}\right)\right)
$$

Also, we provide a formal description of the correlated equilibrium [22]. We denote players as $p=1,2, \ldots, n$. Each player has a strategy $A_{p}$ and we define the strategy profile as $S=\prod_{p=1}^{n} A_{-p}$, where $A_{-p}$ is the profile for all players except q. We define as $\mathrm{y}$ the distribution on A where for $x \in A_{-p}$ we denote by $y_{i, x}$ the probability that a player $\mathrm{p}$ chooses strategy I when all the other players choose $\bar{x}$. The payoff to player $\mathrm{p}$, $u_{i, \bar{x}}^{p}$ for selecting strategy $i \in A$ when everyone selects to play $\mathrm{x}$. The distribution $\mathrm{y}$ is a correlated equilibrium if and only if conditioned on player $\mathrm{p}$ accepting the recommended strategy $i$

$$
\sum_{\bar{x} \in A_{i-p}} u_{i, \bar{x}}^{p} y_{i, \bar{x}} \geq \sum_{\bar{x} \in A_{i-p}} u_{j, \bar{x}}^{p} y_{i, \bar{x}}, \forall i, j \in A_{p}
$$




\section{Game-Theoretic Communication Selection}

Here, we assume that a passing vehicle and the traffic light ahead of it have two communication mediums, an LTE and a DSRC module. In order to accomplish communication, both the vehicle and the traffic light need to select the same medium. The selection is made based on the density of the network and the type of communication, namely safety-critical messages or multimedia download. We will call the traffic light and the vehicle player 1 and 2, respectively, henceforth. Any of the two players could select a medium that suits the most. LTE example shows only the communication from the vehicle to the traffic light. Direct communication between the traffic light and the vehicle can achieve using LTE-V; however, we only take the situation where the base station serves the data. As for the DSRC, direct communication is at play.

Here, we propose using the Battle of the Sexes game model, and similarly, we design a payoff matrix. There are two Nash equilibria in the specific game model in pure strategies, which occurs when both the two players make the same choice (DSRC, DSRC) or (LTE, LTE). Furthermore, there is a mixed strategy equilibrium, when player one is choosing LTE with probability $2 / 3$ and DSRC with probability $1 / 3$ and player two is choosing LTE with probability $1 / 3$ and DSRC with $2 / 3$. The utility will be $(2 / 3$, $2 / 3$ ), which ensures fairness but exhibits lower than the worst outcomes of the Nash equilibria in the pure strategies.

It is suggested at [23] to have a centralized trusted authority that informs the players to select the same outcome. Based on the type of messages exchanged by the players, we see that the players do not have an incentive to change their strategies since it will be worse. The advantage of this process is that the expected rewards are higher $(3 / 2$, $3 / 2$ ) comparing to the Nash equilibrium in the mixed strategies. Formally, we can say that this game has a correlated equilibrium. This suits to the current problem under investigation since we want the two players to select the appropriate strategy depending on the message type.

In the absence of a centralized authority, we formulate the Battle of the Sexes as a potential game, with the potential being:

\begin{tabular}{|c|c|c|}
\hline & LTE & DSRC \\
\hline LTE & 2 & 1 \\
\hline DSRC & 0 & 2 \\
\hline
\end{tabular}

The revenues and costs do not rise with a different selection in this model. On the other hand, there is a good revenue in the same selection by our two players.

\section{Results}

We use the game formulation in [24] for our scenario. The strategies of the players are $\{$ DSRC, LTE , which can be reflected by the utility function values. Each player has her own payoff from a function involving revenues and respective costs. When both players select LTE the revenues are assumed to be $(10,10)$ and the respective costs $(1$, $3)$. If the two players select DSRC the revenues are $(10,20)$ and the costs $(2,5)$. If the 
players select different means, they incur only costs. Note that this could be addressed by the necessity of communication in practical scenarios, i.e., if it is a safety message or a video streaming. The payoff matrix of the proposed game is given below:

\begin{tabular}{|l|c|c|c|}
\hline \multicolumn{2}{|c|}{} & \multicolumn{3}{c|}{ Player 2 } \\
\hline \multirow{4}{*}{ Player 1 1 } & & LTE & DSRC \\
\cline { 2 - 4 } & LTE & $\mathbf{( 9 , 7 )}$ & $(-1,-5)$ \\
\cline { 2 - 4 } & DSRC & $(-2,-3)$ & $\mathbf{( 8 , 1 5 )}$ \\
\hline
\end{tabular}

This game is a weighted potential game with the weights being 1 for player 1 and $3 / 2$ for player 2 . The values of the potential are given below:

\begin{tabular}{|c|c|c|}
\hline & LTE & DSRC \\
\hline LTE & 11 & 3 \\
\hline DSRC & 0 & 12 \\
\hline
\end{tabular}

Player 1 wants to select (LTE, LTE) while player 2 wants (DSRC, DSRC) depending on the type of communication and density, as has been described in a previous section. In particular, when there is knowledge of the players regarding the type of message required, the game will converge to the respective equilibrium.

Furthermore, this game has the finite improvement property whereby no improvement path can be larger than 2 in length. The finite improvement property is based on Theorem 2.15 given in [24]. It refers to a two-player game with two strategies and two equilibria. If a player deviates from a common choice, then the other player will follow by selecting the same strategy (medium), as shown in Fig. 3.

Here player two (the traffic light) selects DSRC and sends the message to player one. Player 1 (the vehicle) could also select DSRC and reach one equilibrium state. However, Player one changes its action to LTE since it requires video transmission, and player two responds by selecting LTE, giving her a higher payoff than if she remained to her DSRC choice. Regardless of the messages, we observe that improvement gets accomplished with two moves. It is pretty easy to see the exchange of messages and the convergence when the vehicle begins the communication first, i.e., Player 1. 


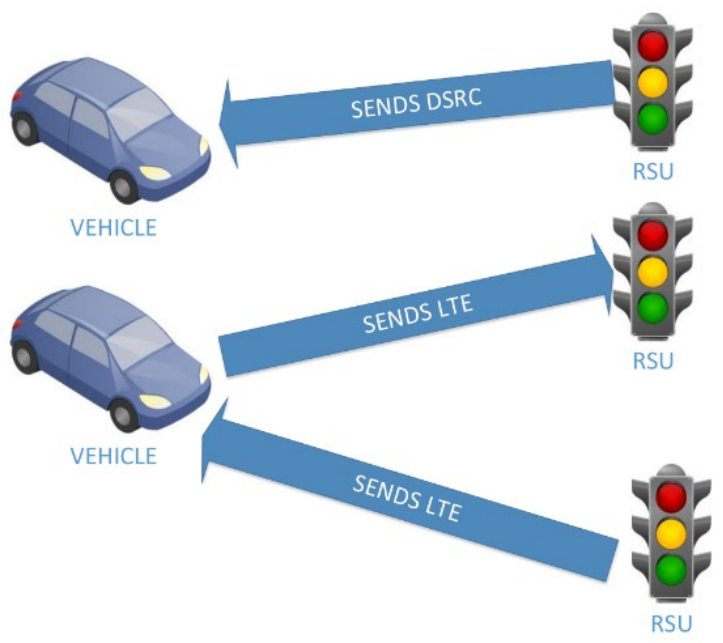

Fig. 3: LTE Selection Example of the Game

Additionally, every potential game has the fictitious play property and converges to equilibrium [25]. This means that there will be two equilibrium points according to the beliefs of the players.

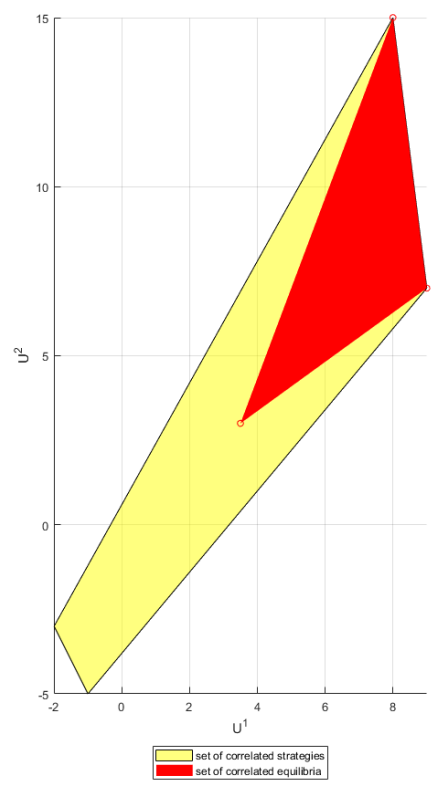

Fig. 4: Set of correlated strategies and correlated equilibria [27]

We also produced the set of correlated equilibria. As we can see in Fig.4, three equilibria are found, namely the two Nash equilibria $(9,7)$ and $(8,15)$ as well as another equilibrium $(3.5,3)$ for Players one and two, respectively. Note that every Nash 
equilibrium is a correlated equilibrium [26]; hence we see that the two equilibria described previously appear on the graph.

\section{Conclusions}

This paper discusses the DSRC and LTE technologies, and it highlights their strengths and weaknesses for ensuring communication among V2I and RSU. An interesting problem is a proper selection between the two technologies.

We propose a game-theoretic formulation to select the most efficient type of communication between a traffic light and a vehicle. This game model has two Nash equilibrium points in the pure strategies and one in the mixed strategies. It is proved that this game exhibits a correlated equilibrium, and it is also formulated as a weighted potential game whereby the centralized authority is absent. Moreover, it is proved that the two players converge to the same medium according to their functionality on the road segment.

It is also essential to highlight the crucial role of the responsible authority in practice since higher efficiency could be achieved by supporting the sustainable development of modern cities that invest in modern technologies to provide higher accuracy, safety, and better quality of life.

Our future work aims to investigate multiple players in this game and examine the behavior of our model.

Acknowledgments. Special thanks to Prof. Bruno Tuffin, author of the book Telecommunication Network Economics: From Theory to Applications, for the valuable conversation regarding the game model approach. This work is funded by the project: "Enhancing research activities of Laboratory of Knowledge and Intelligent Computing" of the Research Committee of the University of Ioannina.

\section{References}

1. Singh, P. K., Nandi, S. K., Nandi, S.: A tutorial survey on vehicular communication state of the art, and future research directions. Vehicular Communications. 18, 100164 (2019).

2. Nshimiyimana, A., Agrawal, D., Arif, W.: Comprehensive survey of V2V communication for 4G mobile and wireless technology. In 2016 International Conference on Wireless Communications, Signal Processing and Networking (WiSPNET). (pp. 1722-1726). IEEE (2016).

3. Whaiduzzaman, M., Sookhak, M., Gani, A., Buyya, R.: A survey on vehicular cloud computing. Journal of Network and Computer applications. 40, 325-344 (2014).

4. Kai, K., Cong, W., Tao, L.: Fog computing for vehicular adhoc networks: paradigms, scenarios, and issues. the journal of China Universities of Posts and Telecommunications. 23(2), 56-96 (2016).

5. Chumkamon, S., Tuvaphanthaphiphat, P., Keeratiwintakorn, P.: The vertical handoff between GSM and ZigBee networks for vehicular communication. In ECTI-CON2010: The 
2010 ECTI International Confernce on Electrical Engineering/Electronics, Computer, Telecommunications and Information Technology. (pp. 603-606). IEEE (2010).

6. Song, Z., Shangguan, L., Jamieson, K.: Wi-Fi goes to town: Rapid picocell switching for wireless transit networks. In Proceedings of the Conference of the ACM Special Interest Group on Data Communication. (pp. 322-334) (2017).

7. Shah, S. A. A., Ahmed, E., Imran, M., Zeadally, S.: 5G for vehicular communications. IEEE Communications Magazine, 56(1), 111-117, (2018).

8. IEEE Standards Association.: Draft Standard for Wireless Access in Vehicular preamble Environments (WAVE)-Multi-channel Operation. IEEE Std 1609.4-2010 (2010).

9. Jiang, D., Delgrossi, L.: IEEE 802.11 p: Towards an international standard for wireless access in vehicular environments. In VTC Spring 2008-IEEE Vehicular Technology Conference. (pp. 2036-2040). IEEE (2008).

10. Shimizu, T., Lu, H., Kenney, J., Nakamura, S.: Comparison of DSRC and LTE-V2X PC5 mode 4 performance in high vehicle density scenarios. In Proc. ITS World Congr. (pp. 1-7) (2019).

11. 3GPP,: Overview of 3GPP Release 8. (2008). http://www.3gpp.org/, Retrieved 2014-03-24.

12. Mir, Z. H., Filali, F.: LTE and IEEE 802.11 p for vehicular networking: a performance evaluation. EURASIP Journal on Wireless Communications and Networking. 2014(1), 115 (2014)

13. Araniti, G., Campolo, C., Condoluci, M., Iera, A., Molinaro, A.: LTE for vehicular networking: a survey. IEEE communications magazine. 51(5), 148-157 (2013).

14. Abboud, K., Omar, H. A., Zhuang, W.: Interworking of DSRC and cellular network technologies for V2X communications: A survey. IEEE transactions on vehicular technology. 65.12 9457-9470 (2016).

15. Vinel, A.: 3GPP LTE versus IEEE 802.11 p/WAVE: Which technology is able to support cooperative vehicular safety applications? IEEE Wireless Communications Letters. 1.2, 125-128 (2012).

16. Xu, Z., Li, X., Zhao, X., Zhang, M. H., Wang, Z.: DSRC versus 4G-LTE for connected vehicle applications: A study on field experiments of vehicular communication performance. Journal of Advanced Transportation. (2017).

17. Von Neumann, J., Morgenstern, O., Kuhn H. W.: Theory of games and economic behavior (commemorative edition). Princeton university press. (2007).

18. Nash Jr, J. F.: The bargaining problem. Econometrica: Journal of the Econometric Society.155-162 (1950).

19. Daskalakis, C., Goldberg, P. W., Papadimitriou, C. H.: The complexity of computing a Nash equilibrium. SIAM Journal on Computing. 39(1), 195-259 (2009).

20. Papadimitriou, C. H.: On the complexity of the parity argument and other inefficient proofs of existence. Journal of Computer and system Sciences. 48.3: 498-532 (1994).

21. Monderer, D., Shapley, L. S.: Potential games. Games and economic behavior. 14(1), 124143 (1996).

22. Saberi, A.: Correlated Equilibria, Lecture Notes, Stanford, (2009).

23. Tardos, E.: Correlated Equilibria. Lecture Notes. (2004).

24. Maillé, P., Tuffin, B.: Telecommunication network economics: from theory to applications. Ca niversity Press (2014).

25. Ewerhart, C., Valkanova, K.: Fictitious play in networks. Games and Economic Behavior. 123, 182-206 (2020).

26. Hart, S., Mas-Colell, A.: A simple adaptive procedure leading to correlated equilibrium. Econometrica, 68(5), 1127-1150 (2000).

27. Iskander (2021). correlated equilibria (https://github.com/ikarib/ce/releases/tag/1.3), GitHub. Retrieved June 9, 2021. 